Roberto Sala

\title{
Die migrationspolitische Bedeutung der italienischen Arbeitswanderung in die Bundesrepublik
}

Die Geschichtswissenschaft unterscheidet meist die „Gastarbeiter“ in der Bundesrepublik aufgrund ihrer nationalen Herkunft voneinander. ${ }^{1}$ Diese Betrachtungsweise erscheint selbstverständlich, beinhaltet aber die Gefahr, Migrationsgeschichte unkritisch als eine Geschichte nationaler Minderheiten zu konzipieren. ${ }^{2} \mathrm{Zu}$ den Aspekten, bei denen die isolierte Analyse des Einwanderungsgeschehens aufgrund der nationalen Herkunft zweckmäßig und erforderlich ist, gehören jedoch unfraglich die migrationspolitischen Rahmenbedingungen. Infolge ihrer Staatsangehörigkeit, die den fassbaren Beleg für die Zugehörigkeit zu einer „Nation“ bildet, fanden die Zuwanderer diverse Spielräume und Zwänge vor, sodass ihre Migrationspfade sehr unterschiedlich ausfallen konnten. In diesem Rahmen sind italienische Arbeitsmigranten von besonderem Interesse, da in der italienischen Zuwanderung drei bedeutende Momente der Massenanwerbung ausländischer Arbeitskräfte vereinigt sind. Zunächst waren die Italiener die ersten „Gastarbeiter“, für die die Bundesrepublik ein Anwerbeabkommen abschloss. Ferner blieben sie mehrere Jahre die einzige Gruppe, die angeworben wurde. Schließlich profitierten nur sie von der Liberalisierung des Arbeitsmarktes innerhalb der Europäischen Wirtschaftsgemeinschaft (EWG).

Dieser Beitrag untersucht die italienische Zuwanderung in die Bundesrepublik vor dem Hintergrund der migrationspolitischen Entwicklung. Wegen seiner Besonderheiten eignet sich der Zustrom italienischer Staatsangehöriger dazu, die spezifischen Auswirkungen des institutionellen Rahmens auf den Migrationsprozess zu verdeutlichen und als Prüfstein für die gesamte westdeutsche „Ausländerpolitik“ zu dienen.

Im ersten Teil wird auf die Genese des deutsch-italienischen Anwerbevertrages von 1955 eingegangen, der als Modell für die späteren Abkommen mit weiteren südeuropäischen Staaten und somit als Beginn der Erwerbsmigration nach Westdeutschland gilt. Im Mittelpunkt stehen hier nicht die unmittelbaren Verhandlungen, die zum Abschluss des Abkommens führten, sondern die Kontinuität der Anwerbung italienischer Arbeitskräfte zwischen „Drittem Reich“ und Bundesrepublik. Im zweiten Teil werden die ersten Jahre der Rekrutierung italienischer Arbeitskräfte thematisiert. Auch wenn diese zunächst einen geringen Umfang aufwies, prägte die Praxis der Anwerbung in Italien in dieser Anfangsphase die spätere Massenanwerbung in Spanien, Griechenland, der Türkei und Jugoslawien mehr, als dies in den bisherigen Forschungen zum Ausdruck kommt. ${ }^{3}$ Im dritten und letzten Teil wird der Aspekt untersucht, der den Zustrom italienischer Arbeitskräfte zum signifikanten Sonderfall der bundesdeutschen Migrationspolitik macht: der Status italienischer Migranten als Angehörige der EWG.

\footnotetext{
${ }^{1}$ Der vorliegende Beitrag ist eine überarbeitete und aktualisierte Fassung des folgenden Aufsatzes: Roberto Sala, Vom „Fremdarbeiter“ zum „Gastarbeiter“. Die Anwerbung italienischer Arbeitskräfte für die deutsche Wirtschaft (1938-1973), in: Vierteljahrshefte für Zeitgeschichte (VfZ) 50 (2007), S.93120.

${ }^{2}$ Hierzu siehe Roberto Sala, Die Nation in der Fremde. Zuwanderer in der Bundesrepublik und nationale Herkunft aus Italien, in: IMIS-Beiträge (2006), H.29, S.99-122.

${ }^{3}$ Johannes-Dieter Steinert ist der Einzige, der über den Anfang der Anwerbung in Italien etwas ausführlicher informiert, wobei er in der Analyse wenig präzise ist; vgl. ders., Migration und Politik. Westdeutschland - Europa - Übersee 1945-1961, Osnabrück 1995, S.284-289.
} 


\section{Kontinuitätslinien}

Hinsichtlich der Genese des „Abkommens über die Anwerbung und Vermittlung von italienischen Arbeitskräften in die Bundesrepublik Deutschland“ vom 20. Dezember 1955 haben lange zwei gegensätzliche Deutungsmuster konkurriert, die am Schärfsten jeweils von Knuth Dohse und Johannes-Dieter Steinert formuliert worden sind. ${ }^{4}$ Nach Dohse ist der Anwerbevertrag als bewusster Schritt der Bundesregierung und speziell des Bundeswirtschaftsministers Ludwig Erhard zu betrachten, der durch die Öffnung des westdeutschen Arbeitsmarkts die Gefahr eines wachstumshemmenden Lohnanstiegs habe bannen wollen. Steinert hingegen verneint den Einfluss arbeitsmarktpolitischer Überlegungen und argumentiert, die Bundesregierung habe durch das Abkommen vor allem Vorteile in den Handelsbeziehungen mit Italien erzielen wollen. Er stützt seine These durch den Beleg, dass die Verhandlungen über den Anwerbevertrag auf eine Initiative der italienischen Regierung zurückgingen und dass Bundesarbeitsminister Anton Storch angesichts der Mitte der 1950er Jahre speziell unter den Vertriebenen noch hohen Arbeitslosigkeit entsprechende Pläne zunächst bekämpft hatte.

Die jüngere Forschung hat verdeutlicht, dass beide Interpretationsansätze defizitär sind, wenn sie als monokausale Erklärungen gelten sollen. ${ }^{5}$ Tatsächlich lässt sich die Genese des Anwerbeabkommens nur verstehen, wenn die Vielfalt der konkurrierenden Umstände berücksichtigt wird. Die Relevanz der Arbeitsmarktpolitik und der internationalen Handelspolitik schließen einander mitnichten aus. ${ }^{6}$ Vielmehr waren Arbeitsmarktpolitik, Handelspolitik und Migrationspolitik einander ergänzende Elemente eines Politikansatzes, der auf eine ältere politische Tradition zurückging. Dies wird deutlich, wenn man die italienische Migrationspolitik vor dem Abkommen von 1955 betrachtet. Die italienische Regierung hatte im ersten Jahrzehnt nach 1945 die staatliche Anwerbung eigener Arbeitskräfte im Ausland zu einem wichtigen Gegenstand internationaler Wirtschaftsverhandlungen gemacht. ${ }^{7}$ Schon 1946 schloss Italien eine Anwerbevereinbarung mit Belgien ab, welche den bedeutenden Einfluss wirtschaftspolitischer Überlegungen aufdeckte: Aufgrund des Abkommens lieferte Belgien für jeden angeworbenen Bergarbeiter eine bestimmte Menge an Kohle, die in Italien besonders knapp war. 1948 bis 1953 folgten Vereinbarungen mit verschiedenen europäischen und außereuropäischen Ländern, unter anderem Frankreich und Argentinien. Die bilateral geregelte Anwerbung wurde in Italien - mit Hinweis auf einen vermeintlich besseren Schutz der Migranten - als „assistierte Auswanderung“ bezeichnet und sollte die Emigration aus dem Lande wieder beleben, die im Faschismus unterbunden worden war. Der Staat sah in der Abwanderung ein Mittel gegen die massive

\footnotetext{
${ }^{4}$ Knuth Dohse, Ausländische Arbeiter und bürgerlicher Staat. Genese und Funktion von staatlicher Ausländerpolitik und Ausländerrecht. Vom Kaiserreich bis zur Bundesrepublik Deutschland, Berlin 1981, S.176-179; Steinert, Migration und Politik, S.220-238.

${ }^{5}$ Vgl. Yvonne Rieker, „Ein Stück Heimat findet man ja immer“. Die italienische Einwanderung in die Bundesrepublik, Essen 2003, S.20, Anm.28; Barbara Sonnenberger, Nationale Migrationspolitik und regionale Erfahrung. Die Anfänge der Arbeitsmigration in Südhessen (1955-1967), Darmstadt 2003, S.60; Grazia Prontera, Partire, tornare, restare? L'esperienza migratoria dei lavoratori italiani nella Repubblica Federale Tedesca nel secondo dopoguerra, Mailand 2009, S.51-59.

${ }^{6}$ Vgl. Karen Schönwälder, Einwanderung und ethnische Pluralität. Politische Entscheidungen und öffentliche Debatten in Großbritannien und der Bundesrepublik von den 1950er bis zu den 1970er Jahren, Essen 2001, S.247f.

${ }^{7}$ Vgl. Rieker, Ein Stück Heimat, S.20. Zur italienischen Migrationspolitik nach 1945 Federico Romero, Emigrazione e integrazione europea 1945-1973, Rom 1991; Andreina De Clementi, Il prezzo della ricostruzione. L'emigrazione italiana nel secondo dopoguerra, Rom/Bari 2010.
} 
Arbeitslosigkeit und für eine Verbesserung der Devisenbilanz, das auch von den Oppositionsparteien und den Gewerkschaften als „notwendiges Übel“ anerkannt wurde. ${ }^{8}$

Die Migrationspolitik der italienischen Republik ist als Ergebnis einer langen und komplexen Entwicklung zu verstehen. Spätestens seit den 1870er Jahren stellte die Massenabwanderung einen zentralen Aspekt italienischer Politik und Geschichte dar. Beeinflusst von Großgrundbesitzern, die einen Lohnanstieg befürchteten, wenn zu viele Arbeitskräfte aus den landwirtschaftlichen Gebieten abwanderten, lehnte der italienische Staat die Massenemigration zunächst ab und versuchte sie einzudämmen. Ein 1888 verabschiedetes Gesetz gewährte jedoch allgemeine Freizügigkeit, woraufhin die Abwanderung als notwendiges Ventil für die inneren sozialen Spannungen anerkannt wurde. ${ }^{9}$ Aber erst zu Beginn des 20.Jahrhunderts entwickelte sich die italienische Abwanderung zum Gegenstand internationaler Politik, insbesondere durch eine Reihe bilateraler Abkommen mit der französischen Regierung.

Die erste französisch-italienische Konvention zur Migrationsfrage wurde 1904 unterschrieben. Sie galt der Verbesserung der sozialen Situation italienischer Arbeitskräfte in Frankreich und diente als Modell für 27 ähnliche bilaterale Verträge, die bis 1914 in ganz Europa abgeschlossen wurden. ${ }^{10}$ Obwohl das primäre Ziel der italienischen Regierung darin bestand, einen besseren Schutz der Migranten zu erreichen, spielten schon bei der ersten Vereinbarung von 1904 auch politische und wirtschaftliche Erwägungen eine wichtige Rolle. 1916 verfestigte sich diese Tendenz durch ein weiteres französisch-italienisches Abkommen, das die direkte Anwerbung von Arbeitskräften in Italien durch französische Behörden vorsah. ${ }^{11}$ Italien verpflichtete sich darin, eine - wenn auch verhältnismäßig kleine - Zahl von Arbeitskräften für die französische Kriegswirtschaft bereitzustellen. Als Gegenleistung musste Frankreich unter anderem für jeden im Bergbau tätigen italienischen Arbeiter eine bestimmte Menge Kohle an Italien liefern - ähnlich wie bei der Anwerbevereinbarung zwischen Italien und Belgien nach dem Zweiten Weltkrieg. Der Historiker Luciano Tosi schreibt zum französisch-italienischen Abkommen von 1916:

„Seitdem war [in Italien] die Auswanderung ins Ausland nicht nur eine Angelegenheit der Arbeiter und der Unternehmer, sondern ein Problem, das durch Verträge zwischen Staaten auszuhandeln war $[\ldots]$. Dies spiegelte eine stark nationalistische Betrachtung der Auswanderung wider, wobei die Emigranten - ohne das Ziel, sie zu beschützen, zu vernachlässigen - als ein Mittel zur Verwirklichung nationaler Interessen wahrgenommen wurden." 12

Im September 1919 schlossen Italien und Frankreich ein weiteres Abkommen, das den sozialen Schutz der Migranten ausbaute und gleichzeitig wirksame Instrumente zur Kon-

\footnotetext{
${ }^{8}$ Vgl. Paola Salvatori, Politica sindacale per l'emigrazione nel secondo dopoguerra, in: Vanni Blengino/Emilio Franzina (Hrsg.), La riscoperta delle Americhe. Lavoratori e sindacato nell'emigrazione italiana in America Latina 1870-1970, Mailand 1994, S.132-146.

${ }^{9}$ Vgl. Maria Rosaria Ostuni, Leggi e politiche di governo nell'Italia liberale e fascista, in: Piero Bevilacqua/Andreina De Clementi/Emilio Franzina (Hrsg.), Storia dell'emigrazione italiana, Bd.1: Partenze, Rom 2001, S.309-319.

${ }^{10}$ Vgl. Luciano Tosi, L'Italia e gli accordi internazionali di emigrazione, in: Centro Studi Emigrazione (Hrsg.), The World in My Hand. Italian Emigration in the World 1860/1960 - Il mondo in mano. L'emigrazione italiana nel mondo 1860/1960, Rom 1997, S.186-195, hier S.187f. Italien unterschrieb 1906 eine neue Abmachung mit Frankreich, 1911 mit Ungarn und 1912 mit Deutschland.

${ }^{11}$ Vgl. Tosi, L'Italia e gli accordi, S.188, Anm.21; Christoph Rass, Bilaterale Wanderungsverträge und die Entwicklung eines internationalen Arbeitsmarktes in Europa, in: Geschichte und Gesellschaft 35 (2009), S.98-134, hier S.112f.

${ }^{12}$ Luciano Tosi, La tutela internazionale dell'emigrazione, in: Piero Bevilacqua/Andreina De Clementi/ Emilio Franzina (Hrsg.), Storia dell'emigrazione italiana, Bd.2: Arrivi, Rom 2002, S.439-456, hier S.443.
} 
trolle der Wanderungsbewegung garantierte. ${ }^{13}$ Die Vereinbarung war - ebenso wie die ab 1917 von den Vereinigten Staaten eingeführten Zuwanderungsbeschränkungen - ein deutliches Zeichen für die zunehmende Kontrolle der Migrationsbewegungen. In den folgenden Jahren schloss Italien mit mehreren Ländern Abkommen zur sozialen Lage der Emigranten ab. Es gelang aber nicht, die radikale Einschränkung von Abwanderungsmöglichkeiten auf globaler Ebene aufzuheben. ${ }^{14}$ Auch die Regierungen der Weimarer Republik widersetzten sich den römischen Plänen, den deutschen Arbeitsmarkt für italienische Staatsangehörige zu öffnen; in Berlin blieb man der neuen restriktiven Migrationspolitik treu. ${ }^{15}$

Ende der 1930er Jahre kehrten sich die Verhältnisse um. Jetzt wollte die deutsche Regierung italienische Arbeitskräfte anwerben. Angesichts einer zunehmenden Arbeitskräfteknappheit unterzeichnete Berlin mit Verbündeten und neutralen Staaten bilaterale Abkommen über einen „Arbeitskräfteaustausch“, wobei aber die italienischen Arbeitskräfte die bei weitem stärkste Gruppe darstellten. ${ }^{16}$ Bis 1942 wurde etwa eine halbe Million italienischer Land- bzw. Industriearbeiter angeworben. Auf beiden Seiten wandelte sich dadurch der Charakter der staatlichen Intervention in die Erwerbsmigration - von der strengen passiven Kontrolle der vergangenen Jahre in eine kontrollierte Förderung.

Bis zum Waffenstillstand Italiens mit den Alliierten im September 1943 bildete diese durch interstaatliche Abkommen „verwaltete Migration“ einen zentralen Gegenstand in den deutsch-italienischen Wirtschaftsbeziehungen, insbesondere bezüglich der Zahlungsbilanz und der Rohstofffrage. Hierbei folgten die Anwerbevereinbarungen zwischen den beiden autoritären Regimen dem von den erwähnten französisch-italienischen Abkommen markierten Pfad, weshalb sich auch die Tendenz verfestigte, die Arbeitsmigration als Mittel nationaler Politik und Gegenstand diplomatischer Verhandlungen zu betrachten. In der Zwischenkriegszeit hatten sich bilaterale Anwerbeverträge als Mittel zur Regulierung eines europäischen Arbeitsmarkts durchgesetzt, wobei sich Frankreich als Einwanderungsland weiterhin als federführend erwies; bahnbrechend war hierbei der franco-polnische Anwerbevertrag von 1919, dem 1927 auch ein deutsch-polnisches Abkommen folgte. ${ }^{17}$ Der Einsatz der Italiener im „Dritten Reich“ zementierte die Tendenz einer zunehmenden Verstaatlichung von Migrationsprozessen: Sämtliche Modalitäten der Anwerbung und der Beschäftigung der italienischen Migranten wurden auf diplomatischer Ebene ausgehandelt und festgeschrieben. ${ }^{18}$ Die Rekrutierung italienischer Arbeitnehmer Ende der 1930er Jahre

\footnotetext{
${ }^{13}$ Es wurde einerseits die von den zwei Staaten „geregelte Auswanderungsfreiheit“ geltend gemacht, anderseits das Prinzip der sozialen Gleichheit von Zuwanderern und Einheimischen; vgl. Tosi, L'Italia e gli accordi, S. 188 .

${ }^{14}$ Nach Tosi, La tutela internazionale, S.445f., wurde die Schließung der ausländischen Arbeitsmärkte gerade durch den Versuch der italienischen Politik beschleunigt, von den Zuwanderungsländern soziale Garantien für die Emigranten zu erhalten.

${ }^{15}$ Vgl. Jochen Oltmer, Migration und Politik in der Weimarer Republik, Göttingen 2005, S.427-433.

${ }^{16}$ Vgl. Brunello Mantelli, „Camerati del Lavoro“. I lavoratori italiani emigrati nel Terzo Reich nel periodo dell'Asse 1938-1943, Florenz 1992; ders., Zwischen Strukturwandel auf dem Arbeitsmarkt und Kriegswirtschaft. Die Anwerbung der italienischen Arbeiter für das „Dritte Reich“, in: Cesare Bermani/Sergio Bologna/Brunello Mantelli, Proletarier der „Achse“. Sozialgeschichte der italienischen Fremdarbeit in NS-Deutschland 1937 bis 1943, Berlin 1997, S.253-390. Deutschland schloss weitere Anwerbeverträge mit Jugoslawien, Ungarn, Bulgarien, den Niederlanden, Kroatien, Spanien und der Slowakei; vgl. Ulrich Herbert, Geschichte der Ausländerpolitik in Deutschland. Saisonarbeiter, Zwangsarbeiter, Gastarbeiter, Flüchtlinge, München 2001, S.125; Hans-Walter Schmuhl, Arbeitsmarktpolitik und Arbeitsverwaltung in Deutschland 1871-2002, Nürnberg 2003.

${ }^{17}$ Vgl. Christoph Rass, Institutionalisierungsprozesse auf einem internationalen Arbeitsmarkt. Bilaterale Wanderungsverträge in Europa zwischen 1919 und 1974, Paderborn 2010.

18 Antonio Dazzi, Accordi tra l'Italia e la Germania in materia di lavoro e assicurazioni sociali 19371942, Tipografia riservata del Ministero degli Affari Esteri, Rom 1942.
} 
stellte somit eine grundlegende Verfestigung der zwischenstaatlich geregelten Massenanwerbung ausländischer Arbeitskräfte dar.

Grundsätzlich handelte es sich um das gleiche System, das die italienische Regierung nach 1945 mit dem Etikett ,assistierte Auswanderung“ zur Anwendung brachte. Zwar gab es Unterschiede zwischen den auf italienischer Seite involvierten Institutionen, was allerdings auf die partielle Zäsur gegenüber dem faschistischen Staatsapparat zurückzuführen ist und nicht auf eine Neuorientierung in der Anwerbungspolitik. Waren vor 1945 die faschistischen Gewerkschaften - die Confederazioni ${ }^{19}$ - und die italienische Botschaft in Berlin zuständig gewesen, übernahmen nun das Arbeitsministerium und die ihm nachgeordneten Arbeitsämter diese Rolle. Auch die anerkannte Migrationshistorikerin Andreina De Clementi hat nahegelegt, dass die Anwerbung italienischer Arbeitskräfte für das „Dritte Reich“ den italienischen Behörden als Vorbild für die nach 1946 abgeschlossenen Anwerbeabkommen diente. ${ }^{20}$ Der von den autoritären Regimen vertretene Dirigismus habe auch im migrationspolitischen Bereich gegolten und großen Einfluss auf die Haltung der italienischen Regierung gegenüber der Auswanderungsfrage nach dem Krieg ausgeübt.

Auf dem Einsatz italienischer Arbeitskräfte im „Dritten Reich“ lastete allerdings der Schatten von Zwangsarbeit und Deportation. Nach dem italienischen „Verrat“ von 1943 waren Hunderttausende italienische Kriegsgefangene und Zivilisten gemeinsam mit Millionen Menschen aus den besetzten Ländern Europas zur Sklavenarbeit in Deutschland gezwungen worden. ${ }^{21}$ Bis 1942 kamen allerdings die italienischen Arbeitskräfte aufgrund der zwischen beiden Staaten abgeschlossenen Vereinbarungen freiwillig in das Deutsche Reich, wo sie als freie Bürger lebten, sofern dies im vom Krieg geprägten Deutschland überhaupt möglich war. ${ }^{22}$ Die freiwillige Rekrutierung ist von der 1943 beginnenden Deportation zur Zwangsarbeit klar zu unterscheiden; die italienische Migrationspolitik nach 1945 konnte an die erste Phase anknüpfen, ohne moralische und politische Bedenken hervorzurufen. ${ }^{23}$

Mit dieser Einschränkung konnte die Rekrutierung italienischer Arbeitskräfte für das „Dritte Reich“ ein Modell für die Anwerbung für die Bundesrepublik nach 1955 bilden, ebenso wie sie ein Vorbild für die gesamte „assistierte Auswanderung“ Italiens in der unmittelbaren Nachkriegszeit dargestellt hatte. ${ }^{24}$ In einem regierungsinternen Brief vom November 1954 wird dies auch offen angesprochen. Es handelt sich um einen Vorschlag

\footnotetext{
${ }^{19}$ Die Confederazione Fascista Lavoratori Agricoltura war für die Landarbeiter, die Confederazione Fascista Lavoratori Industria für die Industriearbeiter zuständig.

${ }^{20}$ Andreina De Clementi, „Curare il mal di testa con le decapitazioni“. L'emigrazione italiana nel secondo dopoguerra. I primi dieci anni, in: „900“ (2003), H.8-9, S.11-27, hier S.13. Vgl. dies., Il prezzo della ricostruzione, S. $6-9$.

${ }^{21}$ Nach dem Waffenstillstand Italiens mit den Alliierten wurden ca. 600000 italienische Soldaten als „Militärinternierte“ eingestuft und zur Zwangsarbeit nach Deutschland deportiert. Vgl. Gabriele Hammermann, Zwangsarbeit für den Verbündeten. Die Arbeits- und Lebensbedingungen der italienischen Militärinternierten in Deutschland 1943-1945, Tübingen 2002.

22 Vgl. Cesare Bermani, Al lavoro nella Germania di Hitler. Racconti e memorie dell'emigrazione italiana 1937-1945, Turin 1998.

${ }^{23}$ Vgl. Livia Novi, Die italienisch-deutsche Anwerbevereinbarung von 1955 im Rahmen der italienischen Wanderungspolitik der fünfziger Jahre, unveröffentlichte Magisterarbeit, Osnabrück 1994, S. 47 .

${ }^{24}$ Die Form der Vereinbarung änderte sich in einem wesentlichen Punkt: Anstelle einer Vielzahl von branchenspezifischen, nach Ablauf von begrenzten Zeiträumen neu verhandelten Verträgen trat ein einziges übergreifendes Abkommen; vgl. Dazzi, Accordi tra l'Italia e la Germania; Accordo fra il Governo della Repubblica Italiana e il Governo della Repubblica Federale di Germania per il reclutamento ed il collocamento della manodopera italiana nella Repubblica Federale di Germania, in: Gazzetta ufficiale della Repubblica italiana, Nr.205 (1956).
} 
des italienischen Schatzministeriums zur Einführung eines zentralisierten Überweisungsdienstes für die Ersparnisse der Emigranten, die in Deutschland arbeiten würden:

„Es ist notwendig, dass die Arbeiter ihr Geld [an die zurückgebliebenen Familien] insgesamt und schnell durch einen systematischen und obligatorischen Kanal nach dem Modell jenes vor dem Krieg schon existierenden [Kanals] nach Italien überweisen können. Damals strömten alle Überweisungen in R. M. der Deutschen Bank zu, die sie [...] der Banca Nazionale del Lavoro überwies, welche wiederum den Gegenwert in italienischen Lire den Familien der Arbeiter auszahlte. Dieses System [...] ersparte [den Migranten] Schwierigkeiten in der Versorgung ihrer Familien in Italien und vermied eine illegale Zerstreuung der Überweisungen. Im Hinblick auf die kommende Auswanderung sollte deshalb die Möglichkeit erwogen werden, zwei große italienische und deutsche Bankinstitute mit einem solchen Uberweisungsdienst zu beauftragen, in Anbetracht der sehr guten Ergebnisse, welche in der Vergangenheit erreicht wurden. ${ }^{425}$

Auch die bundesdeutschen Behörden bezogen sich direkt auf die Anwerbung italienischer Arbeitskräfte im „Dritten Reich“, wie aus einem Bericht ersichtlich wird, den die Deutsche Kommission in Italien zu Beginn ihrer Tätigkeit 1956 verfasste. Der Direktor der Kommission erwähnte darin mehrmals die frühere Rekrutierung und präsentierte sie als erfolgreiches Modell. ${ }^{26}$ Zudem beklagte er gegenüber der Bundesanstalt für Arbeit, dass keiner der im faschistischen Italien eingesetzten deutschen Funktionäre an der neuen Anwerbepraxis beteiligt war - gerade der Verweis auf die mangelnde Kontinuität in der unmittelbaren Personalbesetzung verdeutlicht, dass innerhalb der Bundesanstalt Erfahrungen aus der Rekrutierung italienischer Arbeitskräfte weitergegeben worden waren. ${ }^{27}$ Ein weiterer Hinweis betrifft den Sitz der deutschen Anwerbekommission. Diese siedelte sich zunächst im „Auswanderungszentrum“ in Mailand an, zog aber nach kurzer Zeit nach Verona. ${ }^{28}$ Dort waren bereits vor dem Krieg italienische Arbeitskräfte für die deutsche Wirtschaft angeworben worden.

Die Anwerbung italienischer Arbeitskräfte für die Bundesrepublik ist mithin als Fortsetzung eines Verfahrens zu verstehen, das im Rahmen der „Achse Berlin-Rom“ entstand, zugleich aber auch als das Ergebnis langfristiger internationaler Entwicklungen zur Verstaatlichung der europäischen Migration - wie Christoph Rass in seiner maßgebenden Studie zeigt. ${ }^{29}$ Die italienische Migrationspolitik der ,assistierten Auswanderung“ gab den Anstoß zur Wiederbelebung des bilateral geregelten Anwerbesystems in der Bundesrepublik, das seine Wurzeln in der NS-Zeit hatte und in Berührung mit den pervertierten Facetten des „Fremdarbeitereinsatzes“ gekommen war. Trotz dieses Hintergrunds blieb eine Auseinandersetzung mit der gravierenden Erfahrung von Deportation und Zwangsarbeit, welche Millionen von im „Dritten Reich“ tätige Ausländer prägte, aus. ${ }^{30}$

\footnotetext{
${ }^{25}$ Archivio Centrale dello Stato (im Folgenden: ACS Rom), Presidenza del Consiglio dei Ministri (Vorsitz des Ministerrats), Akte 7 N. 98901, Brief des Schatzministeriums an den Vorsitz des Ministerrats, Überweisungsdienst für die Auswanderer in Deutschland, 30.11.1954. Das vorgeschlagene System fand langfristig keine Anwendung.

${ }^{26}$ Bundesarchiv Koblenz (BArch), B 119/3052, Deutsche Kommission in Italien (DKI), Aktenvermerk, Anwerbung italienischer Arbeitskräfte, 12.4.1956.

${ }^{27}$ Die Bundesanstalt für Arbeit, die für die Anwerbung in Italien verantwortliche deutsche Behörde, war die direkte Nachfolgeinstitution der 1927 gegründeten Reichsanstalt für Arbeitsvermittlung und Arbeitslosenversicherung, die im faschistischen Italien für die Rekrutierung zuständig war.

${ }^{28}$ Vgl. Steinert, Migration und Politik, S. 286.

${ }^{29}$ Rass, Institutionalisierungsprozesse.

${ }^{30}$ Die hier ausgeführte These der Kontinuität in der Anwerbung italienischer Arbeitskräfte zwischen „Drittem Reich“ und Bundesrepublik ist als Bestandteil eines normativ aufgeladenen „Opferplots“ kritisiert worden: Hedwig Richter/Ralf Richter, Der Opfer-Plot. Probleme und neue Felder der deutschen Arbeitsmigrationsforschung, in: VfZ 57 (2009), S.61-97, hier S.73f. Demgegenüber lässt sich festhalten, dass das Hervorheben des Fortbestands von Verwaltungskultur über die Zäsur von 1945
} 


\section{Die Praxis der Anwerbung italienischer Arbeitskräfte}

Gemessen an den Erwartungen staatlicher Stellen erwies sich die Anwerbung italienischer Arbeitskräfte für die Bundesrepublik zunächst als Fehlschlag. Für das Jahr 1956 hatte die deutsche Regierung den italienischen Behörden einen Bedarf von 31000 italienischen Kräften mitgeteilt, wobei es vorwiegend um Saisonkräfte für die Landwirtschaft und das Baugewerbe ging. ${ }^{31}$ Bis zum Jahresende hatte die Deutsche Kommission erst knapp 17000 Arbeitsangebote erhalten und lediglich rund 10000 Arbeitskräfte - davon mehr als die Hälfte für die Landwirtschaft - effektiv anwerben können. ${ }^{32}$ Einerseits wurde der Arbeitskräftebedarf angesichts der wirtschaftlichen Entwicklung in der Bundesrepublik als zu hoch eingeschätzt, andererseits konnten selbst die vorhandenen Anträge wegen bürokratischer Unzulänglichkeiten nicht zügig bearbeitet werden. ${ }^{33}$ Hinzu kam die geringe Anziehungskraft der landwirtschaftlichen Löhne in der Bundesrepublik, sodass viele italienische Interessenten die Angebote ablehnten. ${ }^{34}$

Die wenigen und schlecht bezahlten Arbeitsplätze in der deutschen Landwirtschaft entsprachen mitnichten den Wünschen Italiens, die aber angesichts der deutschen Wirtschaftslage wenig realistisch waren. Für die italienische Regierung bestand das eigentliche Ziel der Abwanderung in die Bundesrepublik in der dauerhaften Beschäftigung von Arbeitskräften außerhalb der Landwirtschaft und in der Ansiedlung der Emigranten im Ausland ${ }^{35}$; nur durch eine „permanente Auswanderung“ ließen sich die strukturellen Ungleichgewichte im Lande und vor allem der Arbeitskräfteüberschuss im Süden abmildern.

Ende 1956 erläuterte der italienische Konsul in Köln die Motive seiner Regierung aus „nationaler Sicht“ und verdeutlichte, dass die „assistierte Auswanderung“ anhand klarer wirtschaftlicher und sozialer Kriterien zu beurteilen war:

„Die tausend italienischen Landarbeiter [in Nordrhein-Westfalen] [...] überweisen an die Familien einige bescheidene Ersparnisse, aber ich glaube nicht, dass sie die Situation derselben dauerhaft verbessern können, denn wegen des saisonalen Charakters ihrer Beschäftigung kann an einen künftigen dauerhaften Einsatz in diesem Lande und demzufolge an eine permanente Auswanderung mit entsprechender Verminderung des demografischen und wirtschaftlichen Drucks nicht gedacht werden. Bedenkt man außerdem die Gesamtkosten der Operation [d. h. der Anwerbung], die nicht nur finanzieller Natur sind [...], entsteht die Frage, ob zumindest unsere Arbeiter von dieser landwirtschaftlichen Auswanderung profitieren." ${ }^{\text {"36 }}$

Andere italienische Konsulate hoben die prekäre Lage der italienischen Landarbeiter in der Bundesrepublik noch stärker hervor. ${ }^{37}$ Nach ihrer Erfahrung hatten mangelhafte Infor-

\footnotetext{
hinaus nicht automatisch auf ein moralisches Urteil hinausläuft, sondern einer nüchternen Betrachtung der historischen Entwicklung entspricht.

31 Anders als bei anderen von Italien abgeschlossenen Anwerbeabkommen enthielt die deutsch-italienische Vereinbarung keine Angaben über den Umfang der Anwerbung. Allerdings hatte die bundesdeutsche Regierung jedes Jahr den geplanten Arbeitskräftebedarf der italienischen Regierung mitzuteilen. Wegen der ständigen Abweichungen zwischen den konkreten Resultaten und den offiziellen Mitteilungen wurde diese Praxis Anfang der 1960er Jahre eingestellt; vgl. Rieker, Ein Stück Heimat, S.24f. ${ }^{32}$ Vgl. Steinert, Migration und Politik, S.284f.

33 Aus deutscher Sicht bestand die größte Schwierigkeit in der Erteilung der Reisepässe durch die italienischen Behörden; ACS Rom, Ministero del Lavoro (ML) 370, Rudolf Petz an Franco Bounous, 24.4. 1956.

${ }^{34}$ Ebenda, Franco Bounous an Rudolf Petz, 7.5.1956.

${ }^{35}$ Ebenda, Italienische Botschaft in der BRD an Ministerium des Auswärtigen, Emigrazione agricola e industriale italiana nella Repubblica federale tedesca, 11.6.1956.

${ }^{36}$ Ebenda, Italienisches Konsulat Köln an Italienische Botschaft, Emigrazione agricola nella RenaniaVestfalia, 14.9.1956.

${ }^{37}$ Ebenda, Italienisches Konsulat Hamburg an Italienische Botschaft, Emigrazione stagionale italiana nella Bassa Sassonia, Amburgo e Brema, 10.9.1956; ebenda, Italienisches Konsulat München an Italieni-
} 
mationen Missverständnisse über die zu erwartende Entlohnung hervorgerufen, sodass viele Arbeitskräfte sich betrogen fühlten. Sie konnten wegen der viel zu niedrigen Löhne keine ausreichende Summe an ihre Familie überweisen und kein Geld für die Zeit der „winterbedingten Arbeitslosigkeit" auf die hohe Kante legen. Außerdem seien die Migranten in sehr kleinen Gruppen oder als Einzelne bei deutschen Kleinbauern untergebracht und würden dementsprechend unter Heimweh und Einsamkeit leiden. Aus Enttäuschung oder gar Verzweiflung hätten zahlreiche Italiener sich dazu entschlossen, Vertragsbruch zu begehen und in die Heimat zurückzukehren. In einigen Gebieten liege die Quote der illegalen Rückkehrer bei über einem Drittel, wobei viele die Konsulate um Hilfe bitten müssten, weil sie sich die Fahrt nach Italien nicht leisten könnten.

Ein Wandel der italienischen Migration in die Bundesrepublik erfolgte erst 1959: Mehr als 40000 italienische Arbeitskräfte kamen nun nach Westdeutschland; drei Fünftel von ihnen waren staatlich angeworben worden. ${ }^{38} 1960$ kam es zum echten Durchbruch, als dank der anhaltend positiven Konjunktur die Zahl der offenen Stellen auf dem deutschen Arbeitsmarkt rasch stieg und mehr als 140000 italienische Arbeitskräfte, davon zwei Drittel über die Deutsche Kommission in Verona, zur Arbeitsaufnahme in die Bundesrepublik zugelassen wurden.

Tabelle 1: Italienische Arbeitswanderung in die Bundesrepublik 1958-1961

\begin{tabular}{lrrrr}
\hline & 1958 & 1959 & 1960 & 1961 \\
\hline Insgesamt & 19398 & 42364 & 141168 & 165793 \\
- über die Deutsche Kommission & 9691 & 25004 & 93284 & 107030 \\
- mit konsularischem Sichtvermerk & 9707 & 17360 & 47884 & 58763 \\
Einschaltungsgrad der Deutschen Kommission & $50,0 \%$ & $59,0 \%$ & $66,1 \%$ & $64,6 \%$ \\
Anteil saisonaler Verträge & $95,0 \%$ & $67,4 \%$ & $45,5 \%$ & $51,8 \%$ \\
\hline
\end{tabular}

Quelle: BA, Anwerbung, Vermittlung, Beschäftigung ausländischer Arbeitnehmer, Erfahrungsbericht 1962, Beilage zu: Amtliche Nachrichten der Bundesanstalt für Arbeitsvermittlung und Arbeitslosenversicherung 4 (1963), S. 34.

1960 begann auch die Anwerbung von Arbeitskräften aus anderen Mittelmeerländern, aber in dieser Phase des Umschwungs stellten die Italiener mit rund 50 Prozent nach wie vor den größten Teil der Arbeitswanderer. Die Deutsche Kommission in Italien hatte sich als ein wirksames und bewährtes Instrument erwiesen, um der ab 1959 erhöhten Nachfrage auf dem Arbeitsmarkt nachzukommen.

Dennoch musste sich die deutsche Arbeitsverwaltung wegen der Anwerbung italienischer Arbeitnehmer mit einigen Grundsatzfragen auseinandersetzen, die 1955 ungelöst geblieben waren. Ein erstes Problem betraf den Umgang mit Saison- bzw. Dauerarbeitskräften, wobei in diesem Zusammenhang mit Dauerarbeitskräften in der Regel jene Zuwanderer gemeint sind, die nicht in einer Saisonbranche beschäftigt waren und über einen einjährigen (verlängerbaren) Vertrag verfügten. Den Erwartungen der Unterzeichner des deutsch-italienischen Abkommens entsprechend bestand die große Mehrheit der nach 1956 angeworbenen italienischen Arbeiter aus Saisonkräften. Diese Tatsache trug entscheidend dazu bei, die Zweifel des Bundesarbeitsministeriums gegenüber dem Abschluss der Vereinbarung zu zerstreuen, da Saisonarbeitskräfte im Falle eines Falles leicht aus dem deutschen Arbeits-

sche Botschaft, Assistenza ai lavoratori italiani in Baviera, 13.9.1956; ebenda, Italienisches Konsulat Stuttgart an Italienische Botschaft, Problemi dell'emigrazione agricola stagionale nel Baden-Württemberg, 11.9.1956.

${ }^{38}$ BA, Anwerbung und Vermittlung ausländischer Arbeitnehmer, Erfahrungsbericht 1961, Beilage zu: Amtliche Nachrichten der Bundesanstalt für Arbeitsvermittlung und Arbeitslosenversicherung 4 (1962), S.25. 
markt zu verdrängen waren. Da die rasch steigende Arbeitskräfteknappheit neben der Bauwirtschaft vorwiegend die Metall- und Textilindustrie betraf, stieg allerdings 1959 der Anteil der „dauerhaft“ beschäftigten Arbeitskräfte auf ein Drittel der insgesamt angeworbenen Italiener, 1960 auf mehr als die Hälfte. ${ }^{39}$

Diese Entwicklung kam - wie die Zunahme der Gesamtzuwanderung - unerwartet und führte zu Befürchtungen, die sich auf erste wirtschaftliche und soziale Folgekosten bezogen. Das baden-württembergische Arbeitsministerium beispielsweise äußerte sich besorgt über das Interesse der italienischen Regierung an einer verstärkten Anwerbung von Dauerarbeitskräften. Im Hintergrund stand die Befürchtung, dass sich die Unternehmen im Falle einer Rezession von einer fest beschäftigten italienischen Belegschaft nicht rasch genug trennen könnten. ${ }^{40}$

Angesichts des wachsenden Arbeitskräftebedarfs verflüchtigten sich solche Zweifel rasch. Stattdessen entwickelte sich eine heftige Kontroverse zwischen der deutschen Arbeitsverwaltung und der saisonabhängigen deutschen Bauwirtschaft wegen der zahlreichen Italiener, die in der Branche beschäftigt waren. Schon im Kaiserreich hatte das deutsche Baugewerbe massiv Arbeitskräfte aus Italien beschäftigt, was die starke italienische Präsenz in dieser Branche auch nach dem Beginn der Anwerbung in den anderen Mittelmeerländern erklären dürfte. Die Schwierigkeiten entstanden, weil deutsche Unternehmen versuchten, ihre italienischen Bauarbeiter über die „warme“ Saison hinaus auch in den Wintermonaten zu beschäftigen. Erste Anzeichen für die ablehnende Haltung deutscher Behörden gegenüber diesem Verhalten gab es bereits 1958, als die Bundesanstalt für Arbeit die Landesarbeitsämter vor dem Einsatz italienischer Bauarbeiter im Stammpersonal bzw. vor deren Beschäftigung im Winter warnte ${ }^{41}$ : Deutsche Arbeitskräfte zu entlassen und italienische zu beschäftigen - das müsse vermieden werden. Im Folgejahr kritisierten deutsche Unternehmen offen diese Vorgabe und 1960 führte der Hauptverband der Deutschen Bauindustrie sogar eine regelrechte „Kampagne“ gegen die Einstellung der Arbeitsverwaltung. ${ }^{42}$ Die Auseinandersetzung erstreckte sich im Laufe der Zeit auf alle ausländischen Bauarbeiter, wobei die Italiener durch ihre Dominanz im Baugewerbe den Hauptgegenstand der Kontroverse bildeten.

Der Plan der Regierung, ähnlich wie bei den polnischen Landarbeitskräften im Kaiserreich eine „Karenzzeit“ für ausländische Bauarbeiter einzuführen, erinnerte an das schweizerische Modell: In der Schweiz waren die in den Saisonbranchen tätigen Ausländer gezwungen, das Land jährlich zu verlassen. ${ }^{43}$ In Westdeutschland konnte aber eine Zwangsrotation der in den empfindlichen Saisonbranchen beschäftigten Ausländer nicht durchgesetzt werden. Ab dem Winter 1961/62 verzichtete die Regierung sogar auf eine

\footnotetext{
${ }^{39}$ BA, Erfahrungsbericht 1961, S. 26.

${ }^{40}$ BArch, B 149/6232, Bundesministerium für Arbeit und Sozialordnung (BMA), Vermerk, Beschäftigung italienischer Arbeiter in der Bundesrepublik, 4.1.1960.

${ }^{41}$ BArch, B 119/3054, Presse-Informationen der BA, Deutsch-italienische Anwerbevereinbarung vom 20.12.1955, hier: Anwerbung von Dauerarbeitskräften für die Wirtschaftssparten, die im deutschitalienischen Protokoll vom 20.12.1955 für die Anwerbung von Saisonkräften vorgesehen sind; ebenda, Ausländergenehmigungsverfahren, hier: Erteilung der Arbeitserlaubnis bei Beschäftigung in Wirtschaftssparten mit Saisoncharakter, 16.10.1958.

${ }^{42}$ BArch, B 149/6232, Presse-Informationen der BA, Saisonbedingte Heimkehr eines Teiles der italienischen Arbeiter, 28.11.1959; ebenda, Hauptverband der Deutschen Bauindustrie an BMA, Auswirkungen des Winterbaues auf die Beschäftigung ausländischer Arbeitskräfte, 3.2.1960; ebenda, BMA, Auswirkungen des Winterbaues auf die Beschäftigung ausländischer Arbeitskräfte, 17.3.1960 und 14.4.1960; ebenda, BA an BMA, Auswirkungen des Winterbaues auf die Beschäftigung ausländischer Arbeitskräfte, 25.5.1960.

${ }^{43}$ Vgl. Hans-Joachim Hoffmann-Nowotny, Switzerland, in: Tomas Hammar (Hrsg.), European Immigration Policy. A Comparative Study, Cambridge 1985, S.206-236.
} 
saisonale Befristung des Aufenthaltes ausländischer Bauarbeiter. ${ }^{44}$ Es fehlten die gesetzlichen Voraussetzungen und der politische Wille für eine andere Regelung, die auch wegen der für 1962 geplanten Liberalisierung der Arbeitsmärkte in der EWG für die Italiener schwer zu realisieren gewesen wäre.

Ein weiterer umstrittener Aspekt, der während der raschen Zunahme italienischer Zuwanderung zutage trat, betraf die Rolle des Staates gegenüber dem Gesamtzustrom ausländischer Arbeitnehmer. Es ging um das Verhältnis zwischen dem Anwerbe- und dem konsularischen Sichtvermerkverfahren. ${ }^{45}$ Der Weg über das Konsulat stellte rechtlich eigentlich den Regelfall dar, während die Anwerbung über die Deutsche Kommission eine Sonderregelung war, die die deutschen und italienischen Behörden getroffen hatten, um die Beschäftigung italienischer Arbeitskräfte in der Bundesrepublik zu begünstigen. Schon im Laufe des Jahres 1959 hatten sich jedoch die Fälle gehäuft, in denen deutsche Konsulate italienischen Bewerbern wegen bürokratischer Unzulänglichkeiten in der Bundesrepublik keine Sichtvermerke mit regulärem Stellenangebot überreichen konnten. Etliche Arbeitsämter hatten sich nämlich geweigert, die zur Erteilung des Sichtvermerks erforderliche Überprüfung des lokalen Arbeitsmarkts durchzuführen, und dies damit begründet, dass alle italienischen Arbeitskräfte sich an die Anwerbekommission wenden müssten. ${ }^{46}$ Ende des Jahres musste das Bundesarbeitsministerium eingreifen:

„Eine Verpflichtung zur Inanspruchnahme der Deutschen Kommission in Italien aufgrund der deutsch-italienischen Anwerbevereinbarung vom 20. Dezember 1955, die hauptsächlich als Institution für die Vermittlung und Besetzung von angebotenen offenen Arbeitsplätzen und die Zusammenstellung von verbilligten Gruppentransporten eingerichtet worden ist, besteht nicht, denn ein solcher Benutzungszwang würde eine Diskriminierung der italienischen Staatsangehörigen gegenüber den Staatsangehörigen aller übrigen Länder bedeuten. “ 47

Trotz dieser Klarstellung blieb die Haltung des Bundesarbeitsministeriums zwiespältig, da sich die beiden Einreisewege überschnitten bzw. weil die Anwerbungskommission die Konsulate in ihrer Aufgabe grundsätzlich ersetzen konnte: Waren die Konsulate für namentliche Anforderungen italienischer Arbeitskräfte durch deutsche Arbeitgeber zuständig, konnte die Deutsche Kommission sowohl bei anonymen als auch bei namentlichen Anforderungen eingeschaltet werden. So verfügte das Bundesarbeitsministerium im September 1960, dass die deutschen Konsulate den Antrag eines italienischen Arbeiters nicht bearbeiten und die Deutsche Kommission informieren sollten, wenn es sich bei dem vorhandenen Arbeitsangebot um eine bereits im Vorjahr belegte Stelle handelte. ${ }^{48}$ Der Grund bestand in der Einführung eines nach Beschwerden der Bauindustrie neu eingeführten Verfahrens innerhalb des Anwerbesystems, das die Neubeschäftigung saisonaler Kräfte beim selben Arbeitgeber vereinfachen sollte. ${ }^{49}$

Diese Maßnahme ist ein deutliches Zeichen dafür, dass spätestens zu diesem Zeitpunkt eine Hierarchie zwischen den beiden Einreisewegen zugunsten des Anwerbesystems ent-

${ }^{44}$ BArch, B 119/3056, BA, Rundschreiben an die Landesarbeitsämter, Beschäftigung nichtdeutscher Arbeitnehmer in der Bundesrepublik Deutschland, hier: Weiterbeschäftigung und Anwerbung von Saisonkräften im Winter 1961/1962, 7.9.1961.

${ }^{45}$ Vgl. Rieker, Ein Stück Heimat, S. 44.

${ }^{46}$ BArch, B 149/6232, Deutsches Konsulat in Neapel an Auswärtiges Amt, Zusicherungen von Aufenthaltserlaubnissen und Erteilung von Sichtvermerken für italienische Arbeiter, die nicht durch die Deutsche Kommission in Verona vermittelt werden, 27.4.1959.

${ }^{47}$ Ebenda, BMA an BA, Einreise italienischer Arbeitnehmer in die Bundesrepublik ohne Inanspruchnahme der Deutschen Kommission in Verona, 4.11.1959.

${ }^{48}$ BArch, B 149/6234, BMA an Deutsche Botschaft und Deutsche Konsulate in Italien, Einreise italienischer Arbeitnehmer in die Bundesrepublik zur Arbeitsaufnahme, 13.9.1960.

${ }^{49}$ BArch, B 149/6234, Hauptverband der Deutschen Bauindustrie, Erteilung von Visa an italienische Arbeitskräfte, 25.4.1960. 
standen war, und nicht zufällig begann die deutsche Verwaltung, die Anwerbung als „Ersten Weg“ und die Einreise mit Sichtvermerk als „Zweiten Weg“ zu bezeichnen. Der „Erste Weg“ wurde ständig verbessert und erweitert, während der „Zweite Weg“ unter erheblichen bürokratischen Hemmnissen und mangelnden Ressourcen litt und sich deshalb als untauglich erwies, um die große Anzahl der Anträge italienischer Arbeitnehmer schnell und effektiv zu bearbeiten. Im Sommer 1961 beklagte das deutsche Konsulat in Palermo die langen Wartezeiten und die vielen Schwierigkeiten, die bei den zur Erteilung des Sichtvermerks erforderlichen Genehmigungen aus der Bundesrepublik auftraten. ${ }^{50}$

Die Kanalisierung und Zentralisierung der Arbeitsmigration durch das Anwerbesystem verlief nicht völlig reibungslos. Das zeigen die Auseinandersetzungen um den „Dritten Weg“, sprich: die Einreise von Ausländern mit einem Touristenvisum, die dann eine Aufenthalts- und Arbeitserlaubnis beantragten. ${ }^{51}$ Noch 1957 hatte die Bundesanstalt für Arbeit die Erteilung einer Arbeitserlaubnis an als „Touristen“ eingereiste Italiener gestattet ${ }^{52}$; dies aber geschah zu einem Zeitpunkt, als die Zuwanderung gering war. Mit der allmählichen Zunahme der Anwerbung wurde diese Zugangsmöglichkeit von der Bundesregierung stark eingeschränkt und 1961 geschlossen, denn die nachträgliche Legalisierung war mit einer zentralisierten Massenanwerbung nicht kompatibel ${ }^{53}$ Dennoch weigerten sich einzelne Bundesländer, diese Vorschrift konsequent umzusetzen. Im Laufe der 1960er Jahre kam es deshalb zu heftigen Kontroversen, aber der „Dritte Weg“ blieb im Vergleich zur Anwerbung eine Ausnahme.

Das Bild einer Verstaatlichungsspirale des westdeutschen Migrationsregimes wird auf den ersten Blick durch die Befunde widerlegt, die Carlos Sanz Díaz zur spanischen Zuwanderung in die Bundesrepublik vorgelegt hat. ${ }^{54}$ Sanz Díaz zeigt, dass es in der ersten Hälfte der 1960er Jahre zu massiven Auseinandersetzungen zwischen deutschen und spanischen Behörden kam. Die Franco-Regierung strebte an, das Anwerbesystem als einzigen Weg für die Abwanderung nach Deutschland durchzusetzen und die Option des konsularischen Sichtvermerks zu verbieten. Diese Haltung erklärt sich dadurch, dass die spanischen Behörden beim „Ersten Weg“ am Rekrutierungsverfahren beteiligt waren und auf das Wanderungsgeschehen Einfluss nehmen konnten, während sie beim „Zweiten Weg“ keine Rolle spielten. Die Bundesregierung verteidigte die Einreise mit konsularischem Sichtvermerk vehement, konnte aber erst nach langjährigen Kontroversen den Streit für sich gewinnen.

Die Verteidigung des „Zweiten Weges“ durch die Bundesregierung steht nicht im tatsächlichen Widerspruch zu der Zentralisierungsdynamik, die am Beispiel der italienischen Zuwanderung deutlich wird. Die Verteidigung des „Zweiten Weges“ darf ferner nicht als Billigung informeller Migrationssysteme missverstanden werden. Das Verfahren mit konsularischem Sichtvermerk gewährleistete eine gewisse Flexibilität, vor allem bei der Einreise von Facharbeitskräften - in Zeiten der Hochkonjunktur hatten die deutschen Behörden kein Interesse, diese Möglichkeit abzuschaffen. In diesem Punkt zeigte die Bundesregierung die Bereitschaft, ein gewisses $\mathrm{Maß}$ an Eigendynamik im Migrationsgeschehen zuzulassen. Dies unterschied die Bundesrepublik von Franco-Spanien, das einen Zentralisierungswahn

\footnotetext{
${ }^{50}$ BArch, B 149/6232, Deutsches Konsulat in Palermo an Deutsche Botschaft in Rom, Arbeitseinsatz sizilianischer Arbeitnehmer in der Bundesrepublik, 13.6.1961.

${ }^{51}$ Vgl. Sonnenberger, Nationale Migrationspolitik, S.82-87; Dohse, Ausländische Arbeiter, S.183.

${ }^{52}$ Vgl. Steinert, Migration und Politik, S.288.

${ }^{53}$ Zur irregulären Zuwanderung in die Bundesrepublik siehe Serhat Karakayali, Gespenster der Migration. Zur Genealogie illegaler Einwanderung in der Bundesrepublik Deutschland, Bielefeld 2008.

${ }^{54}$ Carlos Sanz Díaz, „Illegale“, „Halblegale“, „Gastarbeiter“. Die irreguläre Migration aus Spanien in die Bundesrepublik Deutschland im Kontext der deutsch-spanischen Beziehungen 1960-1973, Berlin 2010.
} 
pflegte, aber es stand für die deutschen Behörden außer Frage, dass der „Zweite“ dem „Ersten Weg“ untergeordnet bleiben sollte.

Ab 1962 wurde - dank der Verordnungen zur Liberalisierung des Arbeitsmarktes in der EWG - gerade für Italiener die Einreise in die Bundesrepublik stark vereinfacht, was innerhalb weniger Jahre der Rekrutierung in Italien fast den Boden entzog. Für Arbeitnehmer aus den übrigen Anwerbeländern behielt das Anwerbesystem seine Zentralität, da bis zum Anwerbestopp von 1973 die Möglichkeit der Einreise über die deutschen Konsulate schließlich doch eingeschränkt wurde. ${ }^{55}$ Dies geschah auch auf Wunsch der Heimatregierungen, deren Arbeitsverwaltungen bei der Anwerbung über große Einflussmöglichkeiten verfügten, während sie an der Ausreise mit Sichtvermerk gar nicht beteiligt waren. ${ }^{56}$

\section{Die Folgen der Freizügigkeit von Arbeitskräften in der EWG}

Durch die nach 1945 abgeschlossenen Anwerbeabkommen konnte Italien nur eine partielle Öffnung ausländischer Arbeitsmärkte erreichen, die weit unter den Erwartungen der Regierung lag. Das Mittel der bilateralen Verträge erwies sich als nicht ausreichend, weil es die durch nationalen Protektionismus bedingte Zuwanderungskontrolle nicht grundsätzlich änderte. ${ }^{57}$ Schon Anfang der 1950er Jahre erklärte deshalb die italienische Regierung die Liberalisierung der Arbeitsmigration zur Bedingung für die Liberalisierung des Handels im Rahmen des europäischen Integrationsprozesses.

Dank dieser Bemühungen stellte die Freizügigkeit der Arbeitnehmer einen wichtigen Gegenstand in den Verhandlungen dar, die im März 1957 zur Gründung der EWG durch Frankreich, Deutschland, Italien, Luxemburg, Belgien und die Niederlande führen sollten. Die deutsch-italienische Vereinbarung von 1955 war das letzte der von Italien abgeschlossenen Anwerbeabkommen und stellte den Endpunkt der Politik der ,assistierten Auswanderung“ dar. Nicht zufällig enthielt der Text des Abkommens den Hinweis, dass seine Bestimmungen einer Liberalisierung der Arbeitsmigration zwischen den europäischen Staaten untergeordnet waren. ${ }^{58}$

Als die Römischen Verträge unterzeichnet wurden, war allerdings die Freizügigkeit der Arbeitskräfte in der EWG noch keine abgemachte Sache. Den Arbeitnehmern wurde nur das pauschale Recht zugestanden, sich innerhalb der Gemeinschaft zur Annahme eines Stellenangebots frei bewegen zu können ${ }^{59}$, wobei die nationalen Regierungen bei hoher Arbeitslosigkeit jederzeit Einschränkungen anordnen konnten. Konkretere Regelungen waren auf nachfolgende Verhandlungen über die Verwirklichung der Freizügigkeit innerhalb der vorgesehenen Übergangszeit von zwölf Jahren verschoben worden. Ein wichtiger Punkt war allerdings schon festgelegt worden. Das von den Niederlanden vertretene Konzept einer interstaatlich geregelten europäischen Arbeitsmarktpolitik konnte sich bei den Gesprächen über den EWG-Gründungsvertrag nicht durchsetzen. Stattdessen wurde -

\footnotetext{
${ }^{55}$ Der „Zweite Weg“ wurde 1965 für männliche Hilfsarbeiter aus den Anwerbeländern und 1973 bis auf wenige Ausnahmen (Familiennachzug, Akademiker, Rückkehrer vom Wehrdienst) verschlossen; vgl. Dohse, Ausländische Arbeiter, S.197.

${ }_{56}$ Vgl. Sonnenberger, Nationale Migrationspolitik, S.74f.

${ }^{57}$ Vgl. Romero, Emigrazione e integrazione, S.39.

58 Artikel 22 des Anwerbeabkommens: Vereinbarung zwischen der Regierung der Bundesrepublik Deutschland und der Regierung der italienischen Republik über die Anwerbung und Vermittlung von italienischen Arbeitskräften nach der Bundesrepublik Deutschland, in: Amtliche Nachrichten der Bundesanstalt für Arbeitsvermittlung und Arbeitslosenversicherung, 25.2.1956.

${ }^{59}$ Vgl. Romero, Emigrazione e integrazione, S. 80.
} 
wenn auch in einer abgeschwächten Form - die deutsche Vorstellung einer allmählichen Aufhebung der Einschränkungen der Arbeitsaufnahme in den Mitgliedstaaten akzeptiert. ${ }^{60}$ Der deutsche Entwurf wurde schon Mitte 1955 in der Konferenz von Messina formuliert, als die deutschen Delegierten von sich aus auf die bislang in der Debatte vernachlässigte Frage der Freizügigkeit eingingen. Dadurch versicherte sich die Bundesregierung sehr wahrscheinlich politischer Solidarität Italiens und verhinderte gleichzeitig andere Vorhaben, die - wie das niederländische - einen weiteren internationalen Eingriff in die interne Arbeitsmarktpolitik vorsahen. ${ }^{61}$

Die Freizügigkeit wurde durch drei Verordnungen vom September 1961, vom März 1964 und vom Oktober 1968 eingeführt. Auf den ersten Blick änderte die erste Verordnung nur wenig an der geltenden Rechtslage, während die beiden folgenden Regelungen die Arbeitsaufnahme für EWG-Angehörige stark vereinfachten.$^{62} 1964$ wurde der Vorrang inländischer Arbeitskräfte aufgehoben, 1968 die Notwendigkeit einer Arbeitserlaubnis gestrichen. Im Rahmen der Hochkonjunktur, die nur 1966/67 kurz aussetzte, und angesichts der entsprechend großzügigen Erteilung der Arbeitserlaubnisse wirkte sich allerdings eine Vorschrift am stärksten aus, die die Verordnung von 1961 begleitete: EWG-Angehörige benötigten ab dem 1.Januar 1962 keinen Sichtvermerk mehr, um in ein anderes Land der Gemeinschaft zur Arbeitsaufnahme einzureisen, sie brauchten nur noch ihren Personalausweis. ${ }^{63}$

Nach 1962 konnten somit italienische Arbeitnehmer faktisch nicht nur bei einem vorhandenen Stellenangebot viel schneller nach Deutschland gelangen, sondern sich auch auf eigene Faust in das Bundesgebiet begeben, um eine Stelle zu suchen. So bestand die Alternative zur staatlichen Anwerbung nicht mehr in einem langwierigen und schwierigen Verfahren bei den deutschen Konsulaten. Anfang der 1970er Jahre kamen nur noch ca. 2 Prozent der italienischen Arbeitskräfte über die Deutsche Kommission in Italien in die Bundesrepublik. Die Anwerbekommission reagierte auf diese Entwicklung mit der verstärkten Rekrutierung qualifizierter Arbeitskräfte, verbuchte aber darin keine Erfolge. ${ }^{64}$

Tabelle 2: Italienische Arbeitswanderung in die Bundesrepublik 1961-1972

\begin{tabular}{lrrrrrr}
\hline & 1961 & 1962 & 1963 & 1964 & 1965 & 1966 \\
\hline Insgesamt & 165793 & 165250 & 134912 & 142120 & 204288 & 165540 \\
- Über die Dt. Kommission & 107030 & 76732 & 31874 & 26537 & 26579 & 13469 \\
- Freie Einreisen & 58763 & 88518 & 103038 & 115583 & 177709 & 152071 \\
Einschaltungsgrad der & $64,6 \%$ & $46,4 \%$ & $23,6 \%$ & $18,7 \%$ & $13,0 \%$ & $8,1 \%$ \\
Deutschen Kommission & & & & & & \\
\hline & & & & & \\
\hline & 1967 & 1968 & 1969 & 1970 & 1971 & 1972 \\
\hline Insgesamt & 58510 & 130236 & 136225 & 168300 & 158725 & 154184 \\
- Uber die Dt. Kommission & 3985 & 10470 & 10206 & 7367 & 4327 & 2092 \\
- Freie Einreisen & 54525 & 119766 & 126019 & 160933 & 154398 & 152092 \\
Einschaltungsgrad der & $6,8 \%$ & $8,0 \%$ & $7,5 \%$ & $4,4 \%$ & $2,7 \%$ & $1,4 \%$ \\
Deutschen Kommission & & & & & & \\
\hline
\end{tabular}

Quelle: Erfahrungsbericht 1972-1973, S.114.

\footnotetext{
${ }^{60}$ Vgl. ebenda, S. 79 .

${ }^{61}$ Vgl. ebenda, S. 69.

${ }^{62}$ Vgl. Heinz Werner, Freizügigkeit der Arbeitskräfte und die Wanderungsbewegungen in den Ländern der Europäischen Gemeinschaft, in: Mitteilungen aus der Arbeitsmarkt- und Berufsforschung 6 (1973), S.326-371.

${ }^{63}$ Vgl. Sonnenberger, Nationale Migrationspolitik, S. 80.

${ }^{64}$ Rieker, Ein Stück Heimat, S.100f.
} 
Der drastische Rückgang der Anwerbung italienischer Arbeitskräfte 1963 löste bei der deutschen Arbeitsverwaltung einen Schock aus. ${ }^{65}$ Zahlreiche Anträge bei der Deutschen Kommission in Italien wurden von den deutschen Arbeitgebern zurückgezogen und an Anwerbestellen in anderen Ländern weitergeleitet, weil italienische Arbeitskräfte kaum mehr zu finden waren. Die Schwierigkeiten bei der Anwerbung in Italien wurden zunächst mit dem Aufschwung der dortigen Wirtschaft begründet, die verstärkt Arbeitskräfte brauchte. ${ }^{66}$ Die Abnahme der italienischen Arbeitswanderung in die Bundesrepublik im selben Jahr verfestigte die Überzeugung, dass eine Erschöpfung der italienischen Arbeitskräftereserven eingetreten sei.

Im Laufe der Zeit erfassten aber die deutschen Arbeitsbehörden die tatsächlichen Hintergründe dieser Entwicklung. ${ }^{67}$ Die italienischen Migranten waren misstrauisch gegenüber Institutionen und verließen sich lieber auf die Informationen von schon ausgereisten Verwandten oder Bekannten, als sich auf eine unbekannte Arbeitsstelle vermitteln zu lassen. 1960 war eine Zweigstelle der Deutschen Kommission in Neapel gegründet worden, um an einem der Brennpunkte der Arbeitslosigkeit präsent zu sein. Auch das half aber nicht viel: Viele Italiener waren nicht zu den zeit- und geldraubenden Reisen und Verfahren bereit, die mit der staatlichen Rekrutierung verbunden waren. Andere hatten Angst vor der strengen Gesundheitsüberprüfung bei der Deutschen Kommission und fürchteten eine Ablehnung, die in ihren Herkunftsgemeinden als Makel empfunden werden würde. ${ }^{68}$ Sogar viele deutsche Unternehmen forderten die Italiener auf, die Deutsche Kommission zu ignorieren, um das langwierige Anwerbeverfahren zu umgehen. ${ }^{69}$

Nach der Einführung der Freizügigkeit in der EWG wurden italienische Arbeitskräfte von Behörden und Arbeitgebern immer häufiger mit einer vermeintlich mangelhaften Disziplin in Verbindung gebracht. Die Italiener stellten unter den „Gastarbeitern“ diejenigen dar, die den höchsten Fluktuationsgrad aufwiesen. Sie wechselten häufig den Arbeitsplatz und zwar ganz gleich, ob der Vertrag ausgelaufen war oder nicht, und kehrten oft in die Heimat zurück. ${ }^{70}$ Obwohl die Arbeitserlaubnispflicht für EWG-Angehörige erst 1968 entfiel, ließ sich schon nach der Aufhebung der Sichtvermerkspflicht 1962 die strenge Kontrolle, die für die anderen Nationalitäten unter den „Gastarbeitern“ durch restriktive migrationspolitische Instrumente galt, bei italienischen Arbeitskräften nicht mehr durchführen. Die Bundesanstalt für Arbeit berichtete, dass wegen der hohen Fluktuation deutsche Arbeitgeber immer mehr dazu neigen würden, keine italienischen Arbeitskräfte mehr zu beschäftigen. ${ }^{71}$

\footnotetext{
${ }^{65}$ BA, Anwerbung, Vermittlung, Beschäftigung ausländischer Arbeitnehmer, Erfahrungsbericht 1963, Beilage zu: Amtliche Nachrichten der Bundesanstalt für Arbeitsvermittlung und Arbeitslosenversicherung 2 (1964), S.10f.

${ }^{66}$ BArch, B 119/3057, DKI, Anwerbung und Vermittlung italienischer Arbeitskräfte nach Deutschland: Entwicklung des Mitwirkungsgrades der Deutschen Kommission, Gründe und Folgerungen, 4.4.1963. ${ }^{67}$ BArch, B 119/3057, DKI, Bericht über die Anwerbung und Vermittlung italienischer Arbeitskräfte für die Bundesrepublik Deutschland, 21.2.1964.

${ }^{68}$ Für die nach Deutschland frei eingereisten Italiener war direkt im Bundesgebiet eine ärztliche Untersuchung vorgeschrieben, die allerdings viel lockerer als die bei der Anwerbungskommission gehandhabt wurde und nur der Ausschließung gravierender oder ansteckender Krankheiten diente.

${ }^{69}$ BArch, B 119/3056, BA an Bundesvereinigung der Deutschen Arbeitgeberverbände, Einreise italienischer Arbeitnehmer nach Deutschland, 26.6.1962.

${ }^{70}$ Die Frage der hohen Fluktuation italienischer Arbeiter in der Bundesrepublik ist komplex und kann hier aus Platzgründen nicht ausgeführt werden. Die Italiener zeigten z. B. Anfang der 1970er Jahre gleichzeitig die höchste Fluktuation und den höchsten Anteil an langfristig beschäftigten Arbeitern; vgl. BA, Repräsentativuntersuchung '72 über die Beschäftigung ausländischer Arbeitnehmer im Bundesgebiet und ihre Familien- und Wohnverhältnisse, Nürnberg 1973.

${ }^{71}$ BArch, B 119/3015, Landesarbeitsamt Nordbayern, Der Präsident, Beschäftigung, Anwerbung und Vermittlung ausländischer Arbeitnehmer, Erfahrungsbericht 1970.
} 
Die italienische Regierung zeigte sich besorgt über diese Entwicklung und klagte, dass zu viele Arbeitnehmer den Sinn der Freizügigkeit „falsch gedeutet“ hätten. ${ }^{72}$ Die systematische Abweichung von der Anwerbung führte aus italienischer Sicht zu einer chaotischen Migration, welche eine politische Planung der Abwanderung unmöglich machte. ${ }^{73}$ Außerdem fürchteten die italienischen Behörden, dass Italiener durch die Rekrutierung in anderen Anwerbeländern verdrängt werden könnten, da die Unternehmer die besser kontrollierbaren Nicht-EWG-Ausländer vorziehen würden. Aus diesen Gründen versuchte die italienische Regierung, einen Primat der EWG-Angehörigen gegenüber Migranten anderer Nationalität durchzusetzen. Dennoch konnte die Bundesrepublik - mit der Unterstützung von weiteren EWG-Staaten - dieses Vorhaben, das zu erheblichen Engpässen in der Anwerbepolitik geführt hätte, erfolgreich abwehren. ${ }^{74}$

Ein „antiitalienisches“ Vorurteil unter den deutschen Arbeitgebern dürfte es tatsächlich gegeben haben, aber vor allem in Bezug auf staatlich rekrutierte Arbeitskräfte. Viele der angeworbenen Italiener begingen nämlich nach kurzer Zeit Vertragsbruch oder tauchten bei der Firma gar nicht auf, weil sie wegen der EWG-Freizügigkeit eine Abschiebung grundsätzlich nicht befürchten mussten. Von der deutschen Arbeitsverwaltung wurde der Verdacht geäußert, diese Arbeiter würden sich mit dem Ziel anwerben lassen, kostenlos in die Bundesrepublik zu gelangen und dort selbstständig eine Arbeit zu suchen. ${ }^{75}$

Gegenüber den frei eingereisten Italienern waren die Vorbehalte viel geringer, wie aus einem Bericht des Landesarbeitsamts in Nordrhein-Westfalen von 1968 ersichtlich ist $^{76}$ :

\begin{abstract}
„Hinsichtlich der Staatszugehörigkeit der ausländischen Arbeitnehmer gibt es in der Bewertung zwar unterschiedliche Beurteilungen [seitens der Arbeitgeber], die aber einer gewissen Objektivität entbehren. Selbst die Kritik an italienischen Arbeitnehmern wegen Nichteinhaltung der Arbeitsverträge und wegen Unbeständigkeit kann nicht verallgemeinert werden. Dagegen spricht nämlich, dass die meistens frei einreisenden italienischen Arbeitnehmer fast ausnahmslos eine Beschäftigung finden und die Italiener die stärkste Ausländergruppe bilden. “77
\end{abstract}

Das Versagen der Anwerbung in Italien nach 1962 überraschte die deutschen Behörden, weil sie die Eigeninitiative italienischer Migranten unterschätzt hatten. Die Abneigung vieler Italiener gegenüber staatlichen Instanzen und Kontrollen war so stark, dass sie manche Bestimmungen selbst dann ablehnten, wenn sie ihnen Schutz boten. Auf Bitten der italienischen Regierung war Anfang der 1960er Jahre die Pflicht eingeführt worden, für alle eingereisten Italiener einen Mustervertrag für die Anwerbung anzuwenden. ${ }^{78}$ Dieser forderte unter anderem von den Arbeitgebern, eine angemessene Unterkunft bereitzustellen. Deutsche Arbeitsämter berichteten erstaunt über die immer heftigeren Beschwerden frei eingereister Italiener, die sich von den Arbeitgebern benachteiligt fühlten, weil der Mustervertrag bei mit Sichtvermerk eingereisten Ausländern nicht erforderlich war. ${ }^{79}$

\footnotetext{
72 Romero, Emigrazione e integrazione, S.109.

${ }^{73}$ Vgl. ebenda, S.110.

${ }^{74}$ Vgl. Rieker, Ein Stück Heimat, S.105-107; Sonnenberger, Nationale Migrationspolitik, S.81f.; Dohse, Ausländische Arbeiter, S.217f.

${ }^{75}$ BArch, B 119/3013, 3014, 3015, 3017, 3018, 3019, Erfahrungsberichte der Landesarbeitsämter, Beschäftigung, Anwerbung und Vermittlung ausländischer Arbeitnehmer, 1966 bis 1970.

${ }^{76}$ Man darf auch nicht vergessen, dass die hohe Fluktuation italienischer Arbeitskräfte bewusst in der Beschäftigungsstrategie von Unternehmen eingesetzt werden konnte; vgl. Anne von Oswald, Volkswagen, Wolfsburg und die italienischen „Gastarbeiter“ 1962-1975. Die gegenseitige Verstärkung des Provisoriums, in: Archiv für Sozialgeschichte 42 (2002), S.55-79.

${ }^{77}$ BArch, B 119/3018, Landesarbeitsamt Nordrhein-Westfalen, Der Präsident, Beschäftigung, Anwerbung und Vermittlung ausländischer Arbeitnehmer, Erfahrungsbericht 1968.

${ }^{78}$ BA, Erfahrungsbericht 1961, S.19.

${ }^{79}$ BArch, B 119/3017, Landesarbeitsamt Nordrhein-Westfalen, Der Präsident, Beschäftigung, Anwerbung und Vermittlung ausländischer Arbeitnehmer, Erfahrungsbericht 1966. Die Beschwerden italieni-
} 
Manche deutsche Beamte erkannten sehr bald, dass neben der Anwerbung auch andere Maßnahmen getroffen werden mussten, sollte es auch nach der Einführung der Freizügigkeit innerhalb der EWG eine Kontrolle der italienischen Zuwanderung geben. Es wurde beispielsweise vorgeschlagen, unverbindliche Beratungsstellen in Süditalien einzurichten, die Informationen über die Deutsche Kommission und über die Stellenaussichten für frei Einreisende vermitteln sollten ${ }^{80}$; der Vorstoß blieb aber ohne Folgen. ${ }^{81}$

Eine Steuerung der neuen italienischen Migrationsbewegung außerhalb des Anwerbesystems wurde nicht einmal versucht. Das erklärt sich zum einen durch die Dominanz der Anwerbung in der damaligen „Ausländerpolitik“, zum anderen durch die deutsche Interpretation der europäischen Freizügigkeit und ihrer Folgen. Die Bundesrepublik hatte einer Liberalisierung der Zuwanderungsregelungen innerhalb der EWG aus politischen Gründen zugestimmt, den „Gastarbeitern“ aber nur eine „negative Integration“ 82 in den deutschen Arbeitsmarkt in Aussicht gestellt. Deutschland hatte zwar die Grenzen für EWGAngehörige geöffnet, ohne allerdings aktive Maßnahmen - weder auf internationaler Ebene noch von Seiten der deutschen Arbeitsverwaltung - für deren Eingliederung zu ergreifen.

\section{Schluss}

Die Anwerbung italienischer Arbeitskräfte macht die Eigendynamik unterschiedlicher Mechanismen der Zuwanderung sichtbar, die sich im Rahmen der häufig als einheitlich beschriebenen „Ausländerpolitik“ entfalteten. Sie stellt einen historischen Sonderfall der Ausländerbeschäftigung in der Bundesrepublik dar, weil sich in ihr der Beginn, die Entwicklung und das frühzeitige Scheitern der Massenrekrutierung ausländischer Arbeitnehmer verschränken. Das deutsch-italienische Anwerbeabkommen von 1955 griff auf eine ältere Verwaltungstradition zurück. Das Anwerbeverfahren kann als Erbe des nationalsozialistischen Deutschlands gelten, es war auch das Ergebnis eines langfristigen Regulierungsprozesses auf europäischer Ebene, der seit Beginn des 20.Jahrhunderts zur internationalen Aushandlung der Zuwanderungsregelungen führte. In der zweiten Hälfte der 1950er und Anfang der 1960er Jahre konnte sich das Anwerbesystem allmählich etablieren, wenn auch nicht ohne Widersprüche. Der mittelfristige Erfolg der Anwerbung in Italien entsprach dem Siegeszug der forcierten Zentralisierung der Arbeitsmigration, welche die gesamte Zuwanderung aus dem Mittelmeerraum nach Westdeutschland prägte. Paradoxerweise scheiterte die Anwerbung nach 1962 aber gerade in Italien. Denn die Einführung der Freizügigkeit in der EWG beseitigte die wichtigste Voraussetzung des Anwerbesystems: die Einschränkung individueller Migrationsfreiheit. Das mag nicht so selbstverständlich sein, wenn man bedenkt, dass bis zum Ersten Weltkrieg die meisten Ausländergruppen ungehemmt ins Kaiserreich zuwandern konnten, mit Ausnahme der polnischen Landarbeitskräfte.

scher Arbeitskräfte führten Ende 1966 zur Abschaffung der Musterverträge; BArch, B 149/22382, Protokoll der Tagung der Deutsch-italienischen Gemischten Kommission, Bonn 23.-26.11.1966.

${ }^{80}$ BArch, B 119/3057, BA, Vermerk des Unterabteilungsleiters zu dem Auftrag, die Hintergründe rückläufiger Anwerbungszahlen in Italien festzustellen, 15.7.1963.

${ }^{81}$ Es wäre genauer zu untersuchen, inwiefern sich die Landesarbeitsämter aktiv mit der Vermittlung frei eingereister italienischer Arbeiter - wie z. B. in Baden-Württemberg 1962 - beschäftigten (BArch, B 119/3056, BA interner Bericht, Freizügigkeit im Rahmen der Europäischen Wirtschaftsgemeinschaft, Mai 1962). Es fehlen jedenfalls Belege für eine gezielte Koordinierung auf Bundesebene zur Vermittlung italienischer Arbeitskräfte.

${ }^{82}$ Vgl. Romero, Emigrazione e integrazione, S. 104. 
Die freie Einreise aus Italien erwies sich für die Bundesrepublik als völlig unlenkbar, als eine unerwünschte Ausnahme im Hinblick auf die europäische Integration. Es war prinzipiell nicht unmöglich, freie Migrationsbewegungen staatlich zu beeinflussen. Aber die Anwerbung hatte sich als Paradigma so stark eingewurzelt, dass alternative Strategien im Kontext staatlicher Planung nicht mehr denkbar erschienen.

Die EWG-Freizügigkeit wirkte sich auch langfristig auf die Präsenz italienischer Migranten in der Bundesrepublik aus. Auf den Ausbruch der Wirtschaftskrise Mitte der 1970er Jahre reagierten die Italiener mit einer deutlich höheren Rückkehrrate als die anderen nationalen Gruppen, da sie bei Verbesserung der Konjunktur wieder in Deutschland eine Arbeit hätten aufnehmen können. ${ }^{83}$ Staatsangehörige aus EWG-Nichtmitgliedstaaten mussten hingegen wegen des Anwerbestopps von 1973 damit rechnen, im Falle einer Rückkehr nicht mehr nach Deutschland einreisen zu dürfen. Vor diesem Hintergrund setzte sich bei italienischen Zuwanderern die zirkuläre Migration fort, die sich bereits im Kontext der hohen Fluktuation während des „Wirtschaftswunders“ abgezeichnet hatte: Ein bedeutender Teil der italienischen Migranten wanderte regelmäßig zwischen Herkunfts- und Zuwanderungsorten, je nach den persönlichen und wirtschaftlichen Chancen und Interessen. Durch Familienzusammenführung und langfristige Niederlassung fand jedoch auch bei Italienern eine Stabilisierung der in Deutschland ansässigen Bevölkerung statt, die seit dem Ende der „Gastarbeiterära“ über eine halbe Million beträgt.

Empirische Daten haben endgültig belegt, dass die italienische Zuwanderung in der Bundesrepublik primär von dem auf den sozialen Bindungen der Herkunftsorte aufbauenden Mechanismus der Kettenwanderung geleitet wurde. ${ }^{84}$ Inwiefern diese Dynamik ausgeprägter als bei anderen nationalen Gruppen war, ist jedoch offen. Die grundsätzliche Bewegungsfreiheit infolge der EWG-Freizügigkeit lässt vermuten, dass sich Kettenwanderung bei Italienern effizienter und uneingeschränkter im Vergleich zu Arbeitsmigranten anderer Staaten auswirken konnte. Es bleibt ein Forschungsdesiderat, zu überprüfen, ob dies zu qualitativen Unterschieden in den Netzwerken der Einwanderer führte.

\footnotetext{
${ }^{83} \mathrm{Zu}$ diesen Aspekten siehe Sonja Haug, Soziales Kapital und Kettenmigration. Italienische Migranten in Deutschland, Opladen 2000.

${ }^{84}$ Vgl. ebenda, S.291f.
} 
